OAI-PMH: http://www.indteca.com/ojs/index.php/Revista Scientific/oai

Ensayo Original / Original Essay

\title{
La Cibersociedad: Modelo por Competencias Digitales y Desafío en la formación Profesional del Profesor Universitario
}

\author{
Autora: Merry Carmen Domínguez Moreno \\ Universidad Nacional Experimental "Rafael María Baralt”, UNERMB \\ merry dominguez@hotmail.com \\ Trujillo, Venezuela \\ https://orcid.org/0000-0003-2422-0983
}

Resumen

El momento presente plantea diferentes y complicados retos en cuanto, a crear diversas profesiones, relacionadas con campos tecnológicos y dada su importancia en la actualidad surge la inquietud de la autora en elaborar el presente ensayo, donde se analizaron posturas argumentadas por la autora referentes a aspectos como desafío emergente en el escenario social, ante la necesidad de una formación basada en competencias, como perspectiva educativa; para cumplir con el deber ser en la formación académicaprofesional en la cultura de la información a partir de la Cibersociedad y competencias digitales en los escenarios universitarios. Concluyendo, es conveniente el modelo basado en competencias para formar profesionales cuyo aprendizaje sea significativo aun en el ámbito en que se desenvuelven los estudiantes basado en el deber ser en la formación académica-profesional en la cultura de la información a partir del modelo por competencias que debe complementarse con la Cibersociedad.

Palabras clave: ciencia y sociedad; competencias del docente; personal académico docente. 


\title{
The Cybersociety: Model for Digital Competencies and Challenge in the Professional Training of the University Professor
}

\begin{abstract}
The present moment poses different and complicated challenges in terms of creating various professions related to technological fields and, given its importance at present, the author's concern arises in the elaboration of the present essay, where postures argued by the author regarding aspects as an emerging challenge in the social scenario, given the need for competencybased training, as an educational perspective; to fulfill the duty to be in the academic-professional formation in the culture of the information from the Cibersociedad and digital competitions in the university scenes. Concluding, it is convenient to use the competency-based model to train professionals whose learning is significant even in the field in which students develop based on the need to be in academic-professional training in the culture of information based on the model for competencies that it must be complemented with the Cybersociety.
\end{abstract}

Keywords: science and society; teacher qualifications; academic teaching personnel. 


\section{Introducción}

El ensayo mostrado, recoge el resultado de una consideración analítica e invectiva de la información temática de la Cibersociedad, donde se realiza una síntesis propia. A la vez, que la autora argumenta manteniendo una actitud crítica hacia el tema objeto de revisión, por considerarla su inquietud personal y desea dar a conocer su relevancia en el mundo actual, donde la sociedad no escapa a los desafíos tecnológicos.

De este modo, el título sugiere una nueva forma de incluir a la Cibersociedad modelo por Competencias Digitales desafío en la formación profesional del profesor universitario. Adjudicando el tráfico de una colectividad en busca de informarse hacia la enfocada al conocimiento, de notificación e ingenio humano. Así, Cibersociedad, ofrece nuevos desafíos para formar estos profesionales universitarios. Ahora bien, hablar de competencias en el profesorado universitario involucra esbozar qué entendemos por competencia en el profesor universitario. En este sentido, se comprende, lo señalado por Escudero (2006):

Son conjunto de valores, creencias y compromisos, conocimientos, capacidades y actitudes que los profesores, tanto a título personal como colectivo... que habrían de adquirir y en las que se ha de crecer para aportar su cuota de responsabilidad a garantizar una buena educación a todos (pág. 89).

Se observa, la alusión que hace el autor referente a aspectos más allá de conocimientos Se hace mención, a aspectos que van dirigidos a reparaciones y contenidos a lograr a favor de optimizar la educación. Asimismo, menciona elementos axiológicos y responsabilidades que alinearán actitudes determinadas de cara al ejercicio de la profesión que sin duda favorecerán la enseñanza a su disposición.

De hecho, pretender edificar nuevas atribuciones orientadas al futuro, ante la mirada de diferentes tendencias, significa brindar aportes reveladores 
al campo universitario, donde se observan problemáticas de acomodamiento a cambios sociales, tecnológicos, culturales en el acontecer de la humanidad. Según, Chiachio, Pievi, Echaverry y Gómez (2009), citados por Castellanos y Castro (2018): son "aspectos que hacen de las universidades un ámbito complicado, a veces conflictivo, inadecuado -en muchos aspectos- y en parte ausente de entusiasmo, con situaciones carentes de apariencias y en búsquedas de nuevos sentidos" (pág. 99).

Eso quiere expresar, la carente motivación universitaria porque no existen mecanismos, estrategias, elementos que ayuden a que estos espacios sociales, de tecnología, de cultura sean capaces de hacer su entrada satisfactoria a estos recintos, además de la escasa búsqueda de herramientas para que estos elementos inunden este ámbito. En ese orden de ideas, la instrucción del profesional universitario, debe estar enfocada hacia la educación del estudiante, donde se muestren interacciones tecno informativas, además de construir conocimientos a favor de difundir y acrecentar las facultades intelectuales según, Pirela y Peña (2005a).

Es el sujeto y sus procesos de aprendizaje los que adquieren centralidad por encima de las tecnologías, las cuales son consideradas como un elemento activador y potenciador de procesos cognitivos. Sobre esta base, se proponen como resultado competencias específicas que debe asumir el profesional de la información en la Cibersociedad (pág. 120).

Estos rasgos en el individuo definen a la Cibersociedad o sociedad actual, de ahí las habilidades en la enseñanza del profesional universitario para incluir como modelo educativo el basado en competencias, porque se busca que el estudiante demuestre habilidades que le sean propias de su entorno y a la vez, le sirvan para su posterior desempeño profesional. Por esto, la relevancia del ensayo presentado porque se busca Analizar las Características de la Cibersociedad como Modelo por Competencias Digitales Desafío en la Formación Profesional del Profesor Universitario. 
Para cumplir con el objetivo se analizarán aspectos como: el desafío emergente en el escenario social, en el trabajo de competencias; conceptualizar el término competencias, características, clasificación; modelo basado en competencias como perspectiva educativa; el deber ser en la formación académica-profesional en la cultura de la información a partir del modelo por competencias; Cibersociedad y competencias digitales en los escenarios universitarios.

\section{Análisis de Planteamientos Teóricos}

En este momento, se plantea un desafío emergente en el escenario social, en el trabajo de competencias en los entornos educativos y en el caso que nos ocupa los universitarios. Por esto, las paulatinas y complejas exigencias de la sociedad posmoderna acontecen, en parte, del avance tecnológico y las innovaciones financieras a las que hoy concurrimos, asignan a las organizaciones educativas y por ende, a sus trabajadores buscar una óptima competitividad.

Por esta razón, hay mayor exigencia en los espacios universitarios aunado a capacitar el talento humano, con propósitos hacia los individuos para que afronten cambios, se adapten rápidamente a nuevas condiciones y así mejorar su desempeño profesional. Estos recientes escenarios sociales emergentes, invitan a asumir con eficacia la instrucción del sujeto en formación caracterizado por ser íntegros, permanentes y con miras a engrandecer los niveles competitivos y productivos del sujeto.

Enfatizando, para lograr los cambios se mencionan a continuación, tres capacidades básicas señaladas por la Organización para la Cooperación y el Desarrollo Económico (OCDE, 2007): cuya misión es promover políticas que mejoren el bienestar económico y social de las personas alrededor del mundo, citada por Ibarra (2002): "la capacidad de innovación, esto es, la imaginación y la creatividad; la capacidad de adaptación, es decir, la adecuación rápida a 
los cambios; y la capacidad de aprendizaje, que es la aprehensión continua y sistemática de nuevos conocimientos" (pág. 55).

Lo señalado, resalta el momento actual donde cobra significación los aportes que puedan reflejar la creatividad e inventiva, adecuarse rápidamente a los cambios sobre todo en los ámbitos sociales que invaden los campos tecnológicos, sin dejar de lado, el proceso cognitivo de la aprehensión incesante ante los nuevos conocimientos que ofrece la Cibersociedad. Al respecto, Morfín (2002), establece:

Esto se debe al hecho de que en la actual coyuntura la generación, transformación y difusión de la información incide notoriamente en todos los aspectos vitales de la sociedad contemporánea, y ello hace que la competitividad sólo pueda crearse y sostenerse a partir de la generación de valor agregado mediante la aplicación del conocimiento humano, que es el único elemento capaz de transformar los recursos naturales en fuentes de riqueza y bienestar (pág. 86).

En palabras del autor, los cambios han adherido transformaciones laborales, o sea, esto solicita de la no observación de fronteras y se observa que los trabajadores ostenten una mezcla de competencias técnicas y académicas que los habiliten para la toma de decisiones, adaptarse fácilmente a la organización del trabajo, además de dilucidar ingeniosamente y revelar destrezas varias. En síntesis, lo ideal sería que este cúmulo de requerimientos sea inserto en el sistema educativo, para que primeramente reconozca las transformaciones para ajustar su disposición y ofrecer formar sujetos capaces de desarrollar habilidades y aprendan por sí mismos. No obstante, Benavides (2002a), señala que las competencias:

Son comportamientos manifiestos en el desempeño laboral que le permiten a una persona actuar eficazmente; también indica que es en esencia un comportamiento productivo observable que engloba los conocimientos, las destrezas, las emociones, factores de personalidad general, amalgamados, desplegados y visibles en la práctica laboral (pág. 18). 
Dicho de otro modo, es la capacidad del sujeto para reubicar, arrastrar sus conocimientos, obteniendo en cada caso altos márgenes de logro donde la evidencia de la posesión de competencias es un ejercicio estable. Asimismo, Fletcher (2000a): las establece "como la aptitud de una persona para desempeñar una misma función productiva en diferentes contextos de trabajo y con base en los resultados esperados" (pág. 35).

En ese orden de ideas, las características esenciales de las competencias, según Malpica (2002a): indica "en primer lugar éstas privilegian el desempeño, por cuanto éste es la expresión concreta de los saberes de un sujeto... conforman una totalidad... y favorecen el desarrollo de mayores niveles de autonomía en los individuos..." (pág. 129).

Ahora bien, sería interesante ajustar el concepto al espacio del aprendizaje, como característica de las competencias. Al respecto, Fletcher (2000b): señala que "una competencia posee tres componentes: el conceptual o el saber; lo procedimental que es el saber hacer y lo actitudinal o el saber ser..." (pág. 42).

De lo señalado, es conveniente señalar con más detalle a qué nos referimos al hablar de cada componente: lo conceptual envuelve todos los componentes de teorías que se deben saber para desempeñar adecuadamente el trabajo. Referente, a lo procedimental o saber hacer significa convertir el conocimiento en acción, operacionalizar los saberes teóricos que se manipulan y lo actitudinal el saber ser que corresponde a la actitud del individuo para efectuar las funciones que le incumben, que contiene la honestidad, autocontrol, dominio de situaciones de alto riesgo, entre otros.

Al mismo tiempo, las competencias se clasifican de acuerdo con varias posturas teóricas, para el presente ensayo la autora escoge la clasificación de Benavides quien las agrupa en tres categorías definidas de la siguiente manera:

Respecto, a las Competencias Básicas, Benavides (2002b): las define 
como "indicadores de desarrollo, porque sirven de fundamento para identificar los logros laborales" (pág. 43). Este tipo de competencia provee de conocer elementalmente el mundo y poder participar socialmente. En este tipo de competencias se pueden agrupar habilidades básicas como la lectura y escritura, realizar operaciones matemáticas, desarrollar el pensamiento creativo, ofrecer soluciones a problemáticas dirigidas a tomar decisiones acertadas al momento de organizar su conceptualización. Además, en estas competencias se incluyen las cualidades personales como autoestima, integridad, autorresponsabilidad, otras.

Asimismo, las Competencias Genéricas: según Benavides (2002c): "se fundamentan en el fortalecimiento de la identidad que nace de las políticas y objetivos organizacionales, que sirven de base para definir las competencias orientadoras" (pág. 43). En este sentido se comprende, que las competencias genéricas son las requeridas en cualquier ámbito profesional y debidamente deben ser transferibles a diferentes contextos de desempeño, dando lugar a la buena organización del empleo, en tal caso, serían los contextos universitarios que sean responsables de su aplicabilidad para su buen funcionamiento.

Las Competencias Específicas, a juicio de Benavides (2002d): "son los comportamientos competitivos característicos frente a productos, servicios, ventas, posicionamientos que hacen referencia al uso y mejoramiento del desempeño personal en la conducción de recursos económicos, científicos, de información, que responden a especialidad laboral de cada individuo" (pág. 45).

Es decir, las competencias específicas, se refieren a las cualidades del sujeto claramente visibles en el sitio de trabajo, debido a comportamientos idóneos se puede lograr un desempeño óptimo y eficaz, a favor de la excelencia institucional. Como observo, en esta clasificación se incentiva el saber hacia espacios delimitados, ya que va de lo general a lo individual. 
El análisis precedente, nos lleva a reflexionar respecto a la vinculación de estas competencias al escenario educativo universitario manejando el término igual como se hace en la industria, donde es de asociar con la indagación de un perfil profesional y valioso para el usuario. Todo lo planteado, se relaciona con los saberes: es decir al conocimiento técnico y de gestión; los haceres 0 destrezas innatas fruto de experiencias y aprendizaje $y$ ontológicamente el ser, referido a aptitudes personales, actitudes, conocimientos, personalidad y valores.

Situación compartida por la autora, debido a que si se relacionan las competencias con lo laboral y profesional se hace con el propósito de optimar la excelencia y eficacia en el desempeño a partir de una instrucción profunda para minimizar lo obsoleto del conocimiento impartido hasta nuestros días.

A la luz de las ideas anteriores, el modelo basado en competencias como perspectiva educativa es sustentado por Malpica (2002b): quien señala que "este concepto es relativamente novedoso y se vincula con el ofrecimiento de un espacio para analizar y construir opciones educativas más acordes con las necesidades individuales y sociales de hoy..." (pág. 134). Parafraseando al autor, este modelo basado en competencias permite afrontar relaciones entre los componentes del contexto y las conveniencias de organización en el trabajo.

En este momento, al abordar la idea educativa fundamentada en las competencias se hace ineludible dilucidar el hecho que en el espacio de las competencias el argumento laboral se coloque al profesional, porque a juicio de Tejada (1999), citado por Pirela y Peña (2005b): estriba en que "la competencia comporta en sí misma conjunto de conocimientos, procedimientos, actitudes, integrados para el ejercicio profesional; así, el dominio de estos saberes hacen al individuo capaz de actuar en situaciones profesionales donde se requiere saber hacer, saber estar" (pág. 125).

En otras palabras, las competencias profesionales son las resultantes 
de la aprehensión que como sujetos adquirimos y desarrollamos en nuestra praxis profesional, infiriendo que para ser un profesional competente necesitamos ser reconocidos como buenos en nuestras prácticas profesionales. Esta postura, es aceptada por Torrado (2000): quien señala "el modelo curricular por competencias demanda reorientar las prácticas educativas, además de revisar la selección y organización de los contenidos y actividades de enseñanza" (pág. 38).

Dicho en otras palabras, es apremiante, o sea se hace necesario descomponer el esquema de cesión individual referentes al conocer, no más aprendizajes memorísticos. Dando así oportunidad para crear espacios experimentales, interpretativos, reflexivos, sobre las temáticas abordadas incluyendo el deber ser en la modificación en contenidos para que sean significativos individual y colectivamente.

De acuerdo con lo expuesto, el modelo educativo basado en competencias busca formar profesionales que internalicen el aprendizaje como procesos flexibles, abiertos y de permanencia en el tiempo y espacio. En síntesis, este modelo integra y combina el aprendizaje académico inmerso en la realidad del profesional universitario para ennoblecer el horizonte de la alineación que reciben los sujetos.

Ahora bien, el deber ser en la formación académica del profesional universitario en la cultura de la información a partir del modelo por competencias, involucra tener presente una sociedad reticular, es decir con estructuras en redes donde se sienta la presencia comunicativa y mediacional. Con el fin de que, se haga visible una cultura que circunscriba representaciones, esparcimientos, organizaciones y productos innovadores que ofrezcan diferentes marcos de desahogo al talento humano.

Se pretende entonces, una alineación basada en competencias para el desarrollo de acciones comunicativas y mediacionales, con soporte en las nuevas tecnologías de la comunicación. Sin embargo, surge la inquietud de 
cuáles serán esas nuevas competencias que según, Pirela y Peña (2005c): "deben desarrollar y aplicar los profesionales de la información frente al desafío y características de la cibersociedad" (pág. 133).

Ante tales planteamientos, la idea subyacente, implica edificar la información supeditada a la del conocimiento. En particular, se intenta crear un profesional que indique convenientemente lo real de este tiempo posmoderno. No obstante, es necesario según, Pirela y Peña, (2005d):

Anexe elementos de innovación y originalidad que favorezcan el replanteamiento de enfoques y tendencias de servicio tradicionales, que si bien respondieron a momentos históricos particulares hoy se muestran agotados y obsoletos ante el avasallante progreso científico y tecnológico que plantea nuevas y complejas necesidades informacionales y cognitivas (pág. 127).

Asimismo, la Cibersociedad debe estar dirigida hacia las competencias digitales en los escenarios universitarios. Un significativo número de autores, como Carrera y Coiduras (2012), citados en San Nicolás, Fariña y Area (2012): "consideran que el desarrollo de la competencia digital en el estudiante, solo será posible si el profesorado cuenta con los conocimientos y el dominio suficiente para incorporarlas en los procesos de enseñanza-aprendizaje que desarrolla" (pág. 230).

Tomando en cuenta, para este ejercicio, según los autores antes citados, se presentan los elementos pertinentes, en la Competencia Digital del profesorado tales como:

a). El conocimiento sobre dispositivos, herramientas informáticas, aplicaciones en red, y capacidad para evaluar su potencial didáctico. Elemento que no puede ser ignorado por el profesor universitario, porque deberá proporcionar una enseñanza-aprendizaje acorde a estos recursos y él habrá de estar capacitado en el manejo y uso de éstos. 
b). Diseñar actividades, situaciones de aprendizaje y evaluación que incorporen las Tecnologías de Información y Comunicación (TIC) de acuerdo con su potencial didáctico, con los estudiantes y con su contexto. En todo ambiente de aprendizaje en este momento, deben ser agregadas las (TIC), el aprendizaje está basado en el manejo de tecnología educativa, con el propósito de adentrar al estudiante en el mundo digital que lidera estos tiempos.

c). La implementación y uso ético, legal y responsable de las (TIC), en el momento de realizar la enseñanza, el profesor deberá conversar acerca de la legalidad existente respecto al uso de la tecnología y apegarse a lineamientos que sustentan ésta como bases legales. Además, de ser ejemplo de ética en el uso y manejo de las TIC, orientarlos cómo tratar estos recursos sin ocasionarle daños a los demás, asumiendo compromisos y responsabilidades.

d). La transformación y mejora de la práctica profesional docente, tanto individual como colectiva. El docente debe estar en constante capacitación, adquirir nuevos conocimientos que transmitirá a los estudiantes, proporcionándoles herramientas para que se haga trabajo en equipo, colaborativo a la vez que los oriente a trabajar individualmente, incentivando la autonomía y el empoderamiento.

e). El tratamiento y la gestión eficiente de la información existente en la red. La reticulación, en los espacios educativos que posean recursos tecnológicos debe estar presente. Las redes al estar actualizadas en cuanto a la información, el alumno aprenderá su conducción de manera más significativa, porque la enseñanza del profesor se hará más relevante debido a que se adapta más a los cambios actuales. Es decir, estarán informados con lo presente.

f). El uso de la red (Internet) para el trabajo colaborativo y la comunicación e interacción interpersonal. Sin duda, usar la red del Internet 
para trabajar colaborativamente y establecer interacciones personales es propio de este mundo cambiante y transformado. Se llega a observar, como en la educación a distancia se trabaja en equipo y colaborativamente.

g). La ayuda proporcionada a los estudiantes para que se apropien de las (TIC) y se muestren competentes en su uso. El tutor, deberá cumplir habilidades inherentes a la aprehensión del estudiante por las (TIC), entre estas actitudes estará el que haya empoderamiento hacia las transformaciones digitales actuales, porque mejorarán notablemente el aprendizaje analógico.

Como se observa, estos autores enfatizan el uso reticular en los elementos que puedan manejar los profesores universitarios en cuanto, al conocimiento y manejo apropiado de todos los recursos tecnológicos. Además de, la inclusión en sus planificaciones de actividades diseñadas para tales fines tomando en cuenta el contexto al que pertenecen.

Acatando, los basamentos legales y su ética en cuanto al manejo y uso de las (TIC). Todo esto, basado en un aprendizaje colaborativo donde se observen actividades individuales y colectivas para optimar las relaciones interpersonales en la consecución de la aprehensión y administración de capacidades en el uso conveniente de las (TIC). De manera concluyente, integrar tecnologías requiere de poseer cantidad y calidad en herramientas técnicas que posean las universidades.

Aunado, al nivel formativo, actual y original que el profesor anexe a los procesos de enseñanza-aprendizaje a desarrollar. Se hace necesario y apremiante que éstos posean y dominen conocimientos en las (TIC), que ayuden a utilizar el potencial creativo y didáctico a la vez que se ofrece al estudiante el empleo de recursos y herramientas que precisan para transformar nuevos conocimientos, en los espacios de la Cibersociedad subyacentes en esta era posmoderna. 


\section{Síntesis del Tópico Objeto de Revisión}

El ensayo mostrado, referente a la Cibersociedad Modelo por Competencias Digitales desafío en la Formación Profesional del Profesor Universitario asigna a los profesionales de la información conducentes a concebir las siguientes conclusiones:

En función de lo argumentado, se concluye que el modelo basado en competencias debe estar situado en la formación de profesionales que imaginen el aprendizaje como un proceso accesible, maleable e imborrable, y que tenga la capacidad traspasar el contexto en el que se recibe la educación formal. Es decir, implementando esta modalidad se integrará y combinarán momentos de aprendizaje académico basados en la objetividad profesional cuya finalidad sea optimizar la instrucción recibida por el estudiante.

Además, el deber ser en la formación académica-profesional en la cultura de la información a partir del modelo por competencias debe complementarse con la Cibersociedad. Por esto, en este momento los diseños educativos se sitúan hacia el desarrollo de modelos de alfabetización tecnológica como espacio para el desarrollo sistémico del profesor universitario, inmersos en realidades culturales y sociales.

En ese orden de ideas, considero es necesario optimizar estas aptitudes digitales, porque involucrarán reformas en los enfoques apoyados en competiciones. Si el profesor universitario utilizara otros materiales y recursos multimedia para la praxis docente podrá desarrollar competencias digitales y además ofrecerá a los estudiantes herramientas actuales adaptados al perfeccionamiento tecnológico y a las pretensiones profesionales que emergen en la actualidad.

\section{Referencias}

Benavides, O. (2002a,b,c,d). Competencias y Competitividad. Diseño para

Organizaciones Latinoamericanas. ISBN: 9584102052; ISBN 13: 
9789584102058. Bogotá, Colombia: McGraw-Hill.

Castellanos, E., \& Castro, J. (2018). Aproximación Teórica para el uso de los Entornos Virtuales en el Proceso de Aprendizaje de los Estudiantes Universitarios. Revista Scientific, 3(7), 99-120. Recuperado de:

https://doi.org/10.29394/Scientific.issn.2542-2987.2018.3.7.5.99-120

Escudero, J. (2006). El Profesor y su Formación: Competencias y Formación Docente al Servicio de un Modelo de Escuela en nuestro tiempo. En Programa y desarrollo temático de Formación y Actualización de la Función Pedagógica. España: Universidad de Murcia, págs. 84-108.

Fletcher, S. (2000a,b). Análisis de Competencias Laborales. México: Panorama.

Ibarra, A. (2002). El Sistema Normalizado de Competencia Laboral. En A. Argüelles (comp.). Competencia laboral y educación basada en normas de competencia. México D.F.: LIMUSA, págs. 25-66.

Malpica, M. (2002a,b). El Punto de Vista Pedagógico. En A. Argüelles (comp.). Competencia laboral y educación basada en normas de competencia. México D.F.: LIMUSA. págs. 123-140.

Morfín, A. (2002). La Nueva Modalidad Educativa: Educación Basada en Normas de Competencia. En A. Argüelles (comp). Competencia laboral y educación basada en normas de competencia. México D.F.: LIMUSA, págs. 81-98.

Organización para la Cooperación y Desarrollo Económicos OCDE (2007). Rapport Annuel 2007. Paris, France: Publications de rOCDE. [Documento en línea]. Recuperado de:

https://www.oecd.org/fr/presse/38528227.pdf

Pirela, J., \& Peña, (2005a,b,c,d). Nuevos Desafíos para la Formación del Profesional de la Información frente al Surgimiento de la 
Cibersociedad: Un Enfoque de Competencias. Investigación bibliotecológica, 19(38), 118-139, ISSN: 2448-8321. [Artículo en línea]. Recuperado de:

http://www.scielo.org.mx/scielo.php?script=sci arttext\&pid=S0187$\underline{358 \times 2005000100007}$

San Nicolás, M., Fariña, E., \& Area, M. (2012). Competencias Digitales del Profesorado y Alumnado en el Desarrollo de la Docencia Virtual. El Caso de la Universidad de la Laguna. Revista Historia de la Educación Latinoamericana, 14(19), 227-245, ISSN: 0122-7238. Recuperado de: http://www.redalyc.org/articulo.oa?id=86926976011 Torrado, M. (2000). Educar para el Desarrollo de las Competencias: una propuesta para Reflexionar. En Universidad Nacional de Colombia (comp). Competencias y proyecto pedagógico. Bogotá: Unibiblos, 3154. 


\section{Merry Carmen Domínguez Moreno}

e-mail: merry dominguez@hotmail.com

Nacida en Venezuela, estado Trujillo. Postgrado en

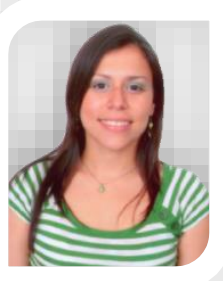

Docencia para la Educación Superior, Universidad Nacional Experimental "Rafael María Baralt" (UNERMB, 2010), Valera, estado Trujillo; Ingeniero de Computación, egresada de la Universidad Valle del Momboy (UVM, 2005), Valera, estado Trujillo. Coordinadora de la Unidad de Sistemas en el Instituto Universitario de Tecnología Mario Briceño Iragorry (IUTEMBI), Carora, estado Lara. (octubre 2005 - abril 2006); Docente Contratada en el Instituto Universitario de Tecnología del estado Trujillo (2010 - 2012). Docente Contratada en la Universidad Politécnica Territorial del Estado Trujillo "Mario Briceño Iragorry", (UPTT “MBI"), desde el año 2010 hasta la actualidad.

El contenido de este manuscrito se difunde bajo una Licencia de Creative Commons ReconocimientoNoComercial-Compartirlgual 4.0 Internacional 